$\Rightarrow$ CORRELATED ELECTRONS

\title{
Interactions on the edge
}

Over the past few years, topological band theory has been very successful in predicting exotic topologically protected states in a variety of material systems. Yet, all this work has been based on the crucial assumption that electron-electron interactions are negligible. In general, this is true, as topological materials have a strong spin-orbit interaction that overrides electron correlations. However, when electrons are strongly confined, correlations can no longer be neglected. Now, two papers in

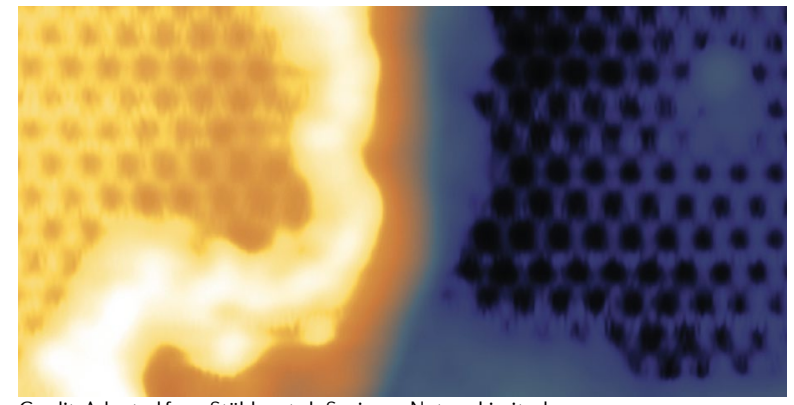

Credit: Adapted from Stühler et al., Springer Nature Limited
Nature Physics explore 1D topological edge states through the lens of Tomonaga-Luttinger liquid (TLL) theory, which describes 1D correlated electron systems.

Typically, 1D edge states, seen in HgTe quantum wells, are topologically protected and do not have strongly correlated electrons. In order to enhance the electron-electron interactions in this system, Jonas Strunz and colleagues brought together two 1D topological edge states (from either side of the quantum well) into a small constriction. Transport measurements revealed that in addition to the expected quantized conductance of such a constriction, an anomalous conduction plateau at half the value of the quantum of conductance is observed. Band structure calculations attribute this anomalous plateau to a correlated scattering process, which can be explained within the TLL theory. The degree of correlation could be controlled by varying the width of the constriction.

Correlated 1D topological states were also observed by Raul Stühler and co-workers in bismuthene, a monolayer of Bi with strong spinorbit coupling. But in this material, the $1 \mathrm{D}$ edge states are far more confined than in most topological systems, owing to the very large band gap of bismuthene. Scanning tunnelling microscopy and spectroscopy studies showed that these narrowly confined 1D states (pictured) displayed classic signatures predicted by TLL theory, such as a linear dispersion relation and the zero-bias anomaly, indicating that strong correlations are also at work in this system.

Ankita Anirban, Associate Editor, Nature Reviews Materials

ORIGINAL ARTICLES Strunz, J. et al. Interacting topological edge channels. Nat. Phys.

https://doi.org/10.1038/s41567-019-0692-4 (2019) | Stühler, R. et al. Tomonaga-Luttinger liquid in the edge channels of a quantum spin Hall insulator. Nat. Phys. https://doi.org/10.1038/ s41567-019-0697-z (2019) 